Article

\title{
Electromagnetic Properties of Graphene-like Films in $\mathbf{K}_{\mathrm{a}}$-Band
}

\section{Sofia Voronovich ${ }^{1}$, Alesya Paddubskaya ${ }^{1}$, Konstantin Batrakov ${ }^{1}$, Polina Kuzhir ${ }^{1,2}$, Sergey Maksimenko ${ }^{1,2}$, Tommi Kaplas ${ }^{3}$ and Yuri Svirko ${ }^{3, *}$}

1 Research Institute for Nuclear Problems of Belarusian State University, Bobruiskaya str., 11, Minsk 220030, Belarus; E-Mails: voronovich_sofiya@bsu.by (S.V.); paddubskaya@gmail.com (A.P.); kgbatrakov@gmail.com (K.B.); polina.kuzhir@gmail.com (P.K.); sergey.maksimenko@gmail.com (S.M.)

2 National Research Tomsk State University, 36, Lenina Avenue, Tomsk 634050, Russia

3 Institute of Photonics, University of Eastern Finland, P.O. Box 111, Joensuu FI-80101, Finland; E-Mail: tommi.kaplas@uef.fi

* Author to whom correspondence should be addressed; E-Mail: yuri.svirko@uef.fi; Tel.: +358-50-3216186.

Received: 25 February 2014; in revised form: 25 April 2014 / Accepted: 29 April 2014 /

Published: 15 May 2014

\begin{abstract}
We studied electromagnetic properties of pyrolytic carbon (PyC) films with thicknesses from $9 \mathrm{~nm}$ to $110 \mathrm{~nm}$. The PyC films consisted of randomly oriented and intertwined graphene flakes with a typical size of a few nanometers were synthesized by chemical vapor deposition (CVD) at $1100{ }^{\circ} \mathrm{C}$ on a quartz substrate. The reflectance and transmittance of these films in $\mathrm{K}_{\mathrm{a}}$-band, 26-37 GHz, were studied both experimentally and theoretically. The discovered remarkably high absorption loss of up to $50 \%$ of incident power, along with chemical stability, makes PyC films attractive for electromagnetic (EM) interference shielding in space and airspace communication systems, as well as in portable electronic devices occupying this frequency slot. Since, in practical applications, the PyC film should be employed for coating of dielectric surfaces, two important issues to be addressed are: (i) which side (front or back) of the substrate should be covered to ensure maximum absorption losses; and (ii) the frequency dependence of absorbance/transmittance/reflectance of binary PyC/quartz structures in the $\mathrm{K}_{\mathrm{a}}$-band.
\end{abstract}


Keywords: microwave frequency range; reflectance; transmittance; absorbance; thin films; pyrolytic carbon; numerical simulation; rectangular waveguide

\section{Introduction}

As the microwave (MW) spectrum becomes more and more crowded, which is synonymous with an increasing electromagnetic (EM) compatibility problem [1], one needs new functional materials for EM coatings, shields and filters, working in specific frequency bands. Such materials should combine high electrical conductivity with good electromagnetic interference (EMI) shielding efficiency (SE), the reciprocal of the transmittance. Small sheet resistance, high optical and IR transparency, ultra-small thickness and ultra-light weight make graphene [2] an excellent candidate for designing such poly-functional materials for EM applications.

Microwave probing has revealed that even a single graphene monolayer is capable of providing almost $20 \%$ of EM attenuation [3]. However, since deposition of graphene on dielectric surfaces of arbitrary shape is still a difficult task, attention of scientists and engineers is currently focused on composite materials filled with graphene nanoplatelets, nanoribbons and flakes that also demonstrate remarkable mechanical, thermal and electromagnetic properties (see [4] and references therein). We have recently suggested an interesting alternative to graphene-based materials by demonstrating that $25 \mathrm{~nm}$ thick pyrolytic carbon (PyC) film, which is morphologically close to the nanographite, being many thousands times thinner than skin depth can attenuate a significant part of incident microwave radiation [5]. In contrast to graphene, PyC films can be easily synthesized not only on metal, but also on dielectric substrate [6] of arbitrary shape which makes it very attractive from the technological point of view. Moreover, being 5-100 times thicker than graphene monolayers, PyC film are much more robust than atom layer thick graphene, i.e., it is hardly influenced by mechanical tension, acoustic waves or an external electric field. The EM response of PyC films can be easily described and characterized in the framework of the conventional techniques allowing quantitative description of their properties. It is also worth noting that coating of a substrate with $\mathrm{PyC}$ film is much cheaper than with graphene, i.e., PyC films are also commercially attractive for a wide range of applications.

The frequency dependence of transmittance/reflectance in $\mathrm{K}_{\mathrm{a}}$-band, 26-37 GHz, for $25 \mathrm{~nm}$ thick PyC film was measured in [5]. An experiment demonstrated surprisingly high absorbance ( 30\%) despite the small sample thickness, which was many thousands times less than the skin depth in metals. The experimental and theoretical description of transmittance/reflectance of PyC films of different thicknesses at the frequency of $30 \mathrm{GHz}$ has been reported recently in our paper [7]. In the current paper, we concentrate on the frequency dependence of absorption, transmission and reflection of PyC films in the $\mathrm{K}_{\mathrm{a}}$-band (26-37 GHz). In particular, we address two important points for practical application: (i) dependence of the absorbance/reflectance of PyC film deposited on quartz substrate on the irradiation effect from the $\mathrm{PyC}$ or substrate side; and (ii) frequency dependence of the EM properties of binary PyC-quartz structures in the $\mathrm{K}_{\mathrm{a}}$-band. 


\section{Experimental Section}

\subsection{PyC Films Fabrication}

PyC films (see Figure 1) were deposited on $0.5 \mathrm{~mm}$ thick quartz substrates by chemical vapor deposition (CVD), described in more detail elsewhere [6,8]. Briefly, the CVD chamber was heated to $700{ }^{\circ} \mathrm{C}$ in hydrogen atmosphere $\sim 10 \mathrm{mBar}$. At the temperature of $700{ }^{\circ} \mathrm{C}$ the hydrogen atmosphere was replaced by $\mathrm{CH}_{4}: \mathrm{H}_{2}$ gas mixture (the $\mathrm{CH}_{4}: \mathrm{H}_{2}$ ratio and pressure are shown in Table 1). Next, in order to start the spontaneous methane decomposition, the CVD chamber was heated up to $1100{ }^{\circ} \mathrm{C}$ by rate of $10{ }^{\circ} \mathrm{C} / \mathrm{min}$. The temperature of the chamber was kept in $1100{ }^{\circ} \mathrm{C}$ for five minutes and then cooled down to $700{ }^{\circ} \mathrm{C}$ during $80 \mathrm{~min}$. At the temperature of $700{ }^{\circ} \mathrm{C}$ the $\mathrm{CH}_{4}: \mathrm{H}_{2}$ gas mixture was replaced by hydrogen. The rest of the cooling was done in hydrogen atmosphere $(\sim 10 \mathrm{mBar})$. It is worth noting that there was no gas flow in the CVD chamber during the deposition process. This allowed us to reduce the gas consumption and also gave more time for polyaromatic hydrocarbon formation and cross linking. However, the change of the temperature during the deposition process was accompanied with change of the pressure in the CVD chamber. After the CVD process both sides of the quartz substrate were covered by the PyC film. In order to characterize the film by microwave measurement, one of the substrate surfaces was cleaned out with the harsh oxygen plasma.

Figure 1. (a) A silica wafer coated uniformly with $\sim 20 \mathrm{~nm}$ thick PyC layer. One can observe that ultrathin carbon film is homogenous over the whole substrate surface; (b) SEM image of the cut PyC film. One can observe that film rips, indicating that it is mechanically strong.
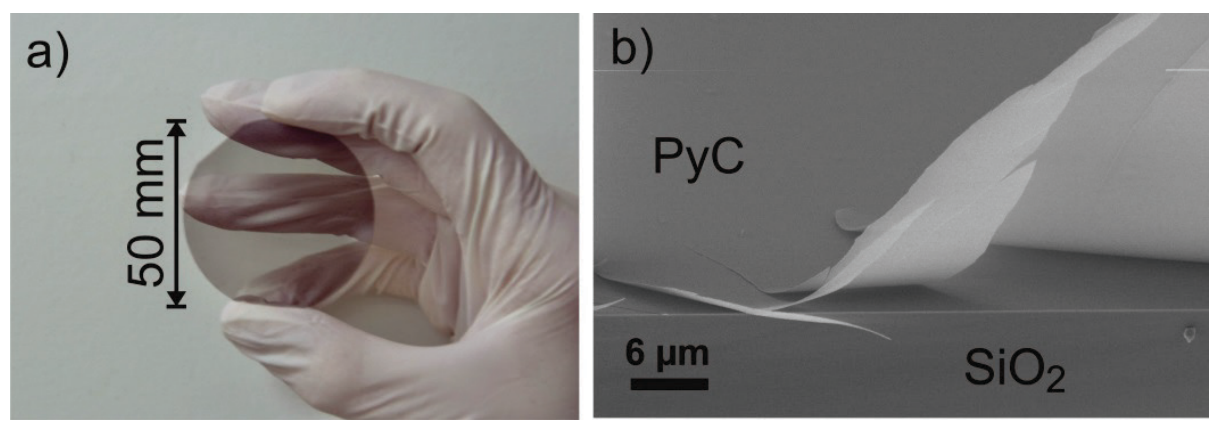

Table 1. PyC films synthesis parameters.

\begin{tabular}{cccccc}
\hline Thickness (nm) & $\mathbf{C H}_{\mathbf{4}}(\mathbf{m L})$ & $\mathbf{H}_{\mathbf{2}} \mathbf{( \mathbf { m L } )}$ & $\begin{array}{c}\text { Pressure at } \\
\mathbf{1 1 0 0}{ }^{\circ} \mathbf{C}(\mathbf{m B a r})\end{array}$ & $\begin{array}{c}\text { Pressure at } \\
\mathbf{7 0 0}{ }^{\circ} \mathbf{C}(\mathbf{m B a r})\end{array}$ & Roughness \\
\hline 9 & 50 & 20 & 22 & 18.0 & 1.08 \\
20 & 75 & 20 & 30 & 24.4 & 1.07 \\
30 & 85 & 20 & 34 & 27.1 & 0.92 \\
75 & 160 & 0 & 53 & 39.0 & 1.25 \\
110 & 180 & 0 & 60 & 45.0 & 1.25 \\
\hline
\end{tabular}

The homogeneity of samples was controlled by SEM (LEO-1455 Vand, Cambridge, UK). PyC films thickness was measured by a stylus profiler (Dektak 150 Veeco Instruments, Plainview, NY, USA) with accuracy of $1.5 \mathrm{~nm}$ and was also controlled by atomic force microscope. The average 
roughness of the films was measured with atomic force microscope (AFM Autoprobe M5, Veeco Instruments, Plainview, NY, USA) for films thinner than $30 \mathrm{~nm}$ and atomic force microscope (Solver P47 PRO NT-MDT, St Petersburg, Russia) for thicker films. One can observe from Table 1 that the roughness of the fabricated PyC films shows a weak dependence on the thickness. The average and root-mean-square roughness were $1.1 \pm 0.2 \mathrm{~nm}$ and $1.5 \pm 0.3 \mathrm{~nm}$, respectively. Raman spectroscopy measurements reported elsewhere [8] revealed that $\mathrm{PyC}$ films produced at our experimental conditions are composed of randomly oriented and intertwined graphene flakes with typical size less than $5 \mathrm{~nm}$. The films also consist of small amounts of amorphous carbon and $\mathrm{sp}^{2}-\mathrm{sp}^{3}$ bonds.

\subsection{Microwave Measurements}

The microwave measurements were provided by scalar network analyzer R2-408R (ELMIKA, Vilnius, Lithuania) at room temperature and normal pressure. The system consists of the sweep generator, waveguide reflectometer, network analyzer and indicator unit (personal computer). Scalar Network Analyzer is designed for measurement of the transmission factor module (attenuation) and reflection factor module (VSWR) of waveguide devices and components in frequency range from $25.96 \mathrm{GHz}$ to $37.5 \mathrm{GHz}$. Frequency sweep bandwidth range can vary from the full frequency range of the instrument to $1500 \mathrm{MHz}$. The set-up provides automatic frequency sweep over the full frequency range with sweep time of $0.08 \pm 0.016 \mathrm{~s}$. Basic error limit of frequency setting does not exceed $\pm 0.2 \%$ in normal conditions. Design of the sweep generator is based on back wave oscillator characteristic to change the generated signal frequency in a wide range depending on voltage change on delay line structure, which is provided by the sweep generator control unit. In the panoramic measurement mode, the generator is operating in periodic frequency sweep from start frequency to end frequency of the sweep range. The output signal is modulated by $100 \mathrm{kHz}$ square pulse modulation. Measurements are based on separation, by the directional couplers, of signals proportional to incident to device under test (DUT) and reflected from DUT mm-wave powers (in case of VSWR measurements) or proportional to incident and passed through DUT (in case of attenuation measurements) mm-waves. The IEC 62431:2008(E) standard specifying the measurement method for the reflectivity of EM materials for the normal incident is used. The EM response of samples as ratios of transmitted/input $\left(\mathrm{S}_{21}\right)$ and reflected/input $\left(\mathrm{S}_{11}\right)$ signals has been measured within $26-37 \mathrm{GHz}$ frequency range $\left(\mathrm{K}_{\mathrm{a}}-\mathrm{band}\right)$. The frequency stability of the oscillator was controlled by frequency meter and was as high as $10^{-6}$. The power stabilization was provided on the level of $7.0 \mathrm{~mW} \pm 10 \mu \mathrm{W}$. Measurement range of EM attenuation was from $0 \mathrm{~dB}$ to $-40 \mathrm{~dB}$. Basic measurement errors of $\mathrm{EM}$ attenuation over the range $0-25 \mathrm{~dB}$ were $\delta\left|\mathrm{S}_{21}\right|= \pm\left(0.6+0.06\left|\mathrm{~S}_{21}\right|\right)$. The substrate before PyC deposition was cut precisely to fit the waveguide cross-section $7.2 \mathrm{~mm} \times 3.4 \mathrm{~mm}$. After standard procedure of the instrument calibration for transmission and reflection measurements, the S-parameters were measured by subsequent insertion of the specimen into the waveguide. The accuracy has been controlled by repetitive measurements.

Reflectance (R), transmittance (T) and absorbance (A) are obtained from the measured S-parameters in the following way: $R=S_{11}^{2}, T=S_{21}^{2}, A=1-R-T$. The scheme of waveguide measurements is presented in Figure 2.

We performed measurements in both "quartz + PyC" (the EM wave incidents on the quartz substrate) and "PyC + quartz" (the EM wave incidents on the PyC film) geometries. 
Figure 2. The waveguide measurements setup in the "quartz + PyC" geometry.

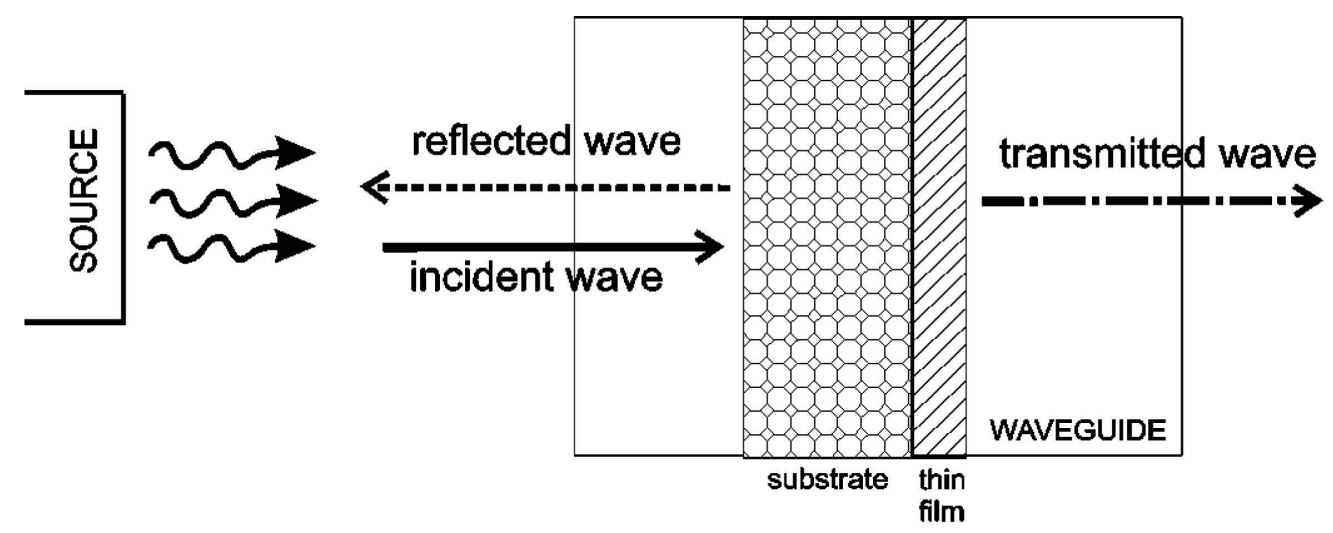

\section{Theory}

The longitudinal component of magnetic field associated with a TE waveguide mode is described by the following equation:

$$
H_{z i}=i b_{i}^{ \pm} \cos \left(k_{x} x\right) \cos \left(k_{y} y\right) \exp \left( \pm i K_{i} z\right)
$$

where + and - correspond to the waves propagating along waveguide axis $z$ in positive and negative directions, respectively, subscript $i=0,1,2,3$ labels vacuum, substrate, PyC film and vacuum, respectively, $x, y$ are cross sectional coordinates of the rectangular waveguide, $k_{x}=\frac{\pi n}{a}, k_{y}=\frac{\pi m}{b}$, where $a, b$ are the lateral waveguide dimensions, $n, m$ are integers, $K_{i}=\sqrt{k^{2} \varepsilon_{i}-\left(\frac{\pi n}{a}\right)^{2}-\left(\frac{\pi m}{b}\right)^{2}}$ is the longitudinal wavenumber in the $i$-th medium, $b_{i}$ is mode amplitudes in $i$-th medium to be found from boundary conditions. In particular, if the waveguide supports $\mathrm{TE}_{1,0}$ mode, $n=1, m=0$ and $K_{i}=\sqrt{k^{2} \varepsilon_{i}-\left(\frac{\pi}{a}\right)^{2}}$.

Let us consider the "quartz + PyC" geometry when the EM wave is incident on the quartz substrate. Boundary conditions in this geometry have the following form [7]:

$$
\left\{\begin{array}{c}
b_{0}^{+}+b_{0}^{-}=b_{1}^{+}+b_{1}^{-} \\
K_{0}\left(b_{0}^{+}-b_{0}^{-}\right)=K_{1}\left(b_{1}^{+}-b_{1}^{-}\right) \\
b_{1}^{+} e^{i K_{1} l_{1}}+b_{1}^{-} e^{-i K_{1} l_{1}}=b_{2}^{+}+b_{2}^{-} \\
K_{1}\left(b_{1}^{+} e^{i K_{1} l_{1}}-b_{1}^{-} e^{-i K_{1} l_{1}}\right)=K_{2}\left(b_{2}^{+}-b_{2}^{-}\right) \\
b_{2}^{+} e^{i K_{2} l_{2}}+b_{2}^{-} e^{-i K_{2} l_{2}}=b_{3}^{+} \\
K_{2}\left(b_{2}^{+} e^{i K_{2} l_{2}}-b_{2}^{-} e^{-i K_{2} l_{2}}\right)=K_{0} b_{3}^{+}
\end{array}\right.
$$

Here $l_{1}$ and $l_{2}$ are the thicknesses of substrate and PyC film, respectively. By solving Equation (2) under assumptions $K_{2} l_{2} \ll<, \operatorname{Im}\left\{\varepsilon_{2}\right\}>1$ and $\left|K_{2}\right|^{2}>K_{1} K_{0}$ we arrive at the following equations for the complex transmission $\left(t=b_{3}^{+} / b_{0}^{+}\right)$and reflection $\left(r=b_{0}^{-} / b_{0}^{+}\right)$coefficients in the “quartz + PyC" geometry: 


$$
\begin{aligned}
& t_{q p}= T_{0} \frac{e^{i \phi}}{1+\delta-\sqrt{R_{0}}\left(\sqrt{R_{0}}+\delta\right) e^{2 i \phi}} \\
& r_{q p}=\frac{\sqrt{R_{0}}(1+\delta)-\left(\sqrt{R_{0}}+\delta\right) e^{2 i \phi}}{1+\delta-\sqrt{R_{0}}\left(\sqrt{R_{0}}+\delta\right) e^{2 i \phi}}
\end{aligned}
$$

where $\phi=K_{1} l_{1}$ is the phase accrued by the wave after one pass through the substrate, $R_{0}=\left|K_{1}-K_{0}\right|^{2} /\left|K_{1}+K_{0}\right|^{2}$ and $T_{0}=4 K_{1} K_{0} /\left|K_{1}+K_{0}\right|^{2}$ are reflectance and transmittance of the vacuum-substrate interface, respectively, $\delta=l_{2} / l_{\sigma}$ where $l_{\sigma}=c^{2}\left(K_{0}-K_{1}\right) / 4 \pi \omega \sigma$ is the characteristic length that describe EM properties of the two-layer structure.

In order to clarify the meaning of $l_{\sigma}$ it is instructive to consider the interaction of the EM with a free standing thin conductive film. In such a case the amplitude reflection/transmission coefficients can be obtained from Equation (2) at $K_{0}=K_{1}$ and $l_{1}=0$. By substituting $T_{0}=1$ and $R_{0}=\phi=0$ into Equation (3) we arrive at the following equations for the transmittance and reflectance of the conductive film with thickness $l_{2}$ :

$$
\begin{aligned}
& T=\frac{l_{\sigma}^{2}}{\left(l_{2}+l_{\sigma}\right)^{2}}, \\
& R=\frac{l^{2}}{\left(l_{2}+l_{\sigma}\right)^{2}}, \\
& A=1-T-R=\frac{2 l_{2} l_{\sigma}}{\left(l_{2}+l_{\sigma}\right)^{2}}
\end{aligned}
$$

One can observe from Equation (4) that at $l_{2}=l_{\sigma}$ absorption losses in the film are maximum and $A=0.5$.

In the "PyC + quartz" geometry, the amplitude transmission coefficient (and, hence, transmittance) is expectedly the same as that in the "quartz + PyC" geometry. However the complex reflection coefficients (and hence reflectance and absorbance) are different in "quartz + $\mathrm{PyC}$ " and "PyC + quartz" geometries:

$$
r_{p r}=\frac{\sqrt{R_{0}}-\delta-\sqrt{R_{0}}(1-\delta) e^{2 i \phi}}{1+\delta-\sqrt{R_{0}}\left(\sqrt{R_{0}}+\delta\right) e^{2 i \phi}}
$$

Thus one can conclude that overall shielding efficiency, which is determined by the transmittance, does not depend on which side of the substrate the absorbing coating is deposited. However the reflectance and hence absorbance of such two-layer structure do depend on the geometry chosen.

It also follows from Equation (5) that at a finite substrate thickness, the transmittance and reflectance is affected by the interference of the EM wave in the substrate. In our experimental conditions, we cannot expect strong oscillations in the reflected and transmitted waves because the phase accrued by the wave after one pass through the substrate varies from $0.05 \pi$ to about $0.1 \pi$ over the studied spectral range. However the interference effects can strongly influence the amount of the reflected and absorbed EM energy because it is determined by the EM wave amplitude inside the PyC film. 


\section{Results and Discussion}

In the numerical calculations the following parameters were used: permittivity of substrate $\varepsilon_{1}=3.5$, PyC film conductivity $\sigma=4.5 \times 10^{14} \mathrm{~s}^{-1}\left(5.0 \times 10^{4} \mathrm{~S} / \mathrm{m}\right)$, lateral waveguide dimensions are $a=7.2 \mathrm{~mm}, b=3.4 \mathrm{~mm}$.

The measured and calculated reflectance, transmittance and absorbance are presented in Figure 3 for films being $75 \mathrm{~nm}$ and $110 \mathrm{~nm}$ thick and in Table 2 for thinner films (9 nm, $20 \mathrm{~nm}$ and $30 \mathrm{~nm}$ ).

Comparison of experimental data with results of calculations in the frequency range $26-37 \mathrm{GHz}$ shows the metal-like behavior of PyC. One can notice that in the "PyC + quartz" geometry, $110 \mathrm{~nm}$ thick PyC film is capable to absorb not more than $40 \%$ of incident power, whereas in the "quartz + PyC" geometry, the absorption losses are as high as $50 \%-55 \%$. Thus the latter geometry can be employed to improve sensitivity of the detectors of MW radiation.

Figure 3. Calculated (solid lines) and measured (circles, squares and triangles) reflectance, transmittance and absorbance spectra of $110 \mathrm{~nm}(\mathbf{a 1}, \mathbf{a 2})$ and $75 \mathrm{~nm}(\mathbf{b 1}, \mathbf{b 2})$ thick films in the "PyC + quartz" (a1, b1) and "quartz + PyC" (a2, b2) geometries.

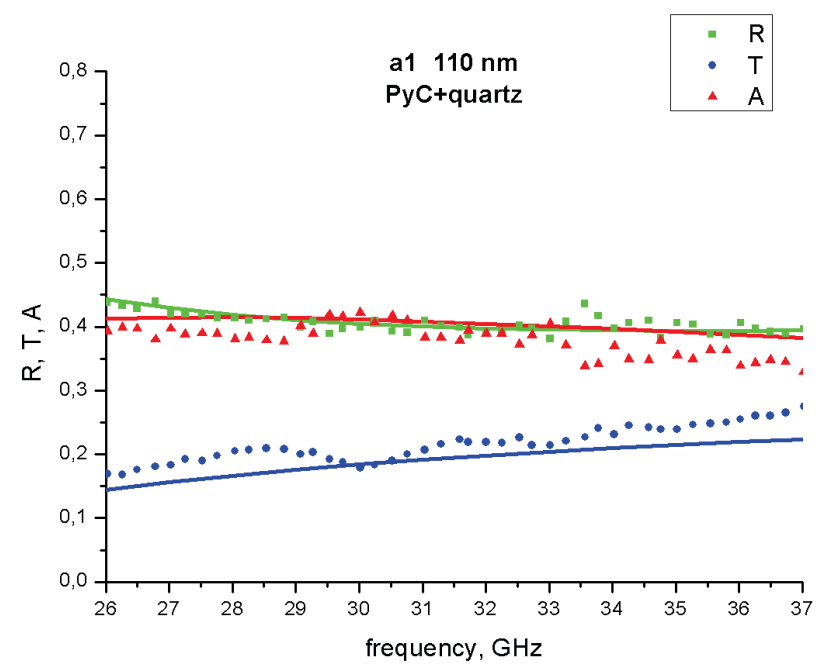

(a1)

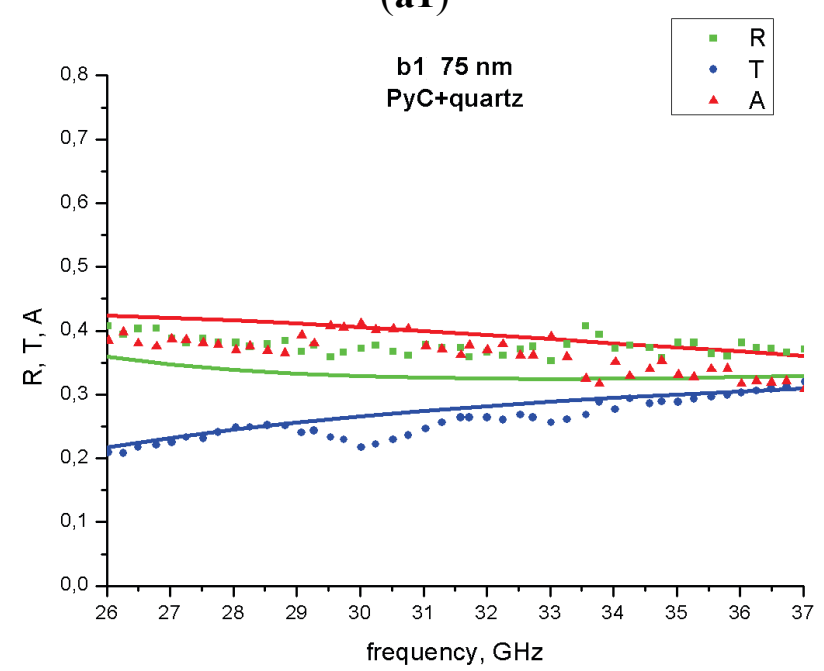

(b1)

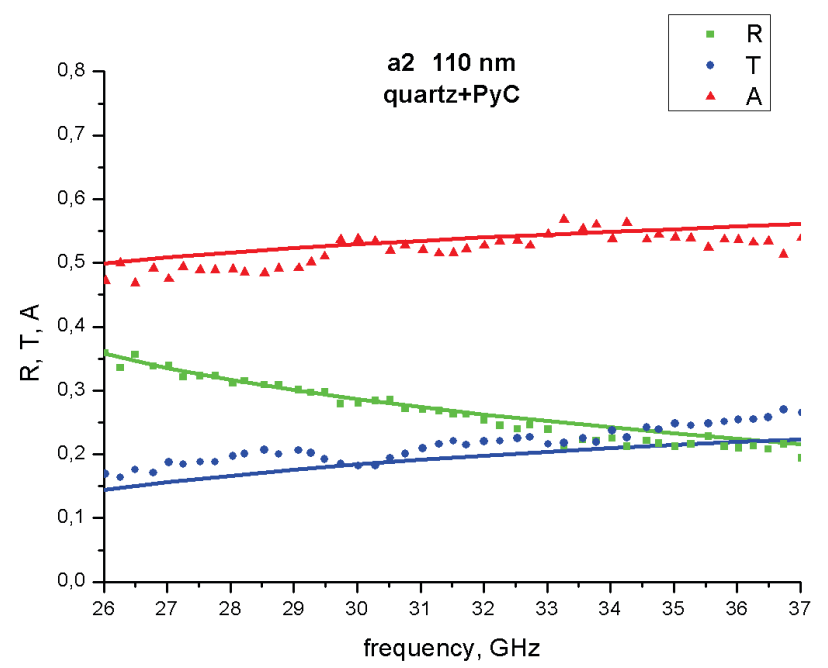

(a2)

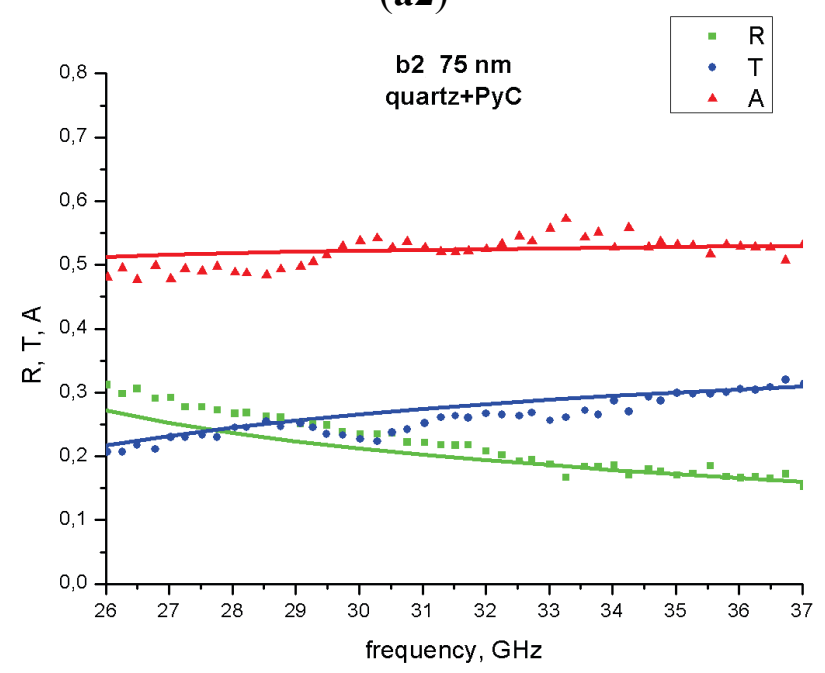

(b2) 
Table 2. Theoretical (subscript "th") and experimental (subscript "exp") transmittance, reflectance and absorbance for thinnest PyC films on $\mathrm{SiO}_{2}$ substrate.

\begin{tabular}{ccccccc}
\hline \multirow{2}{*}{ Frequency, GHz } & \multicolumn{7}{c}{ PyC Thickness, 9 nm } \\
\cline { 2 - 7 } & $\boldsymbol{R}_{\exp }$ & $\boldsymbol{R}_{\text {th }}$ & $\boldsymbol{T}_{\text {exp }}$ & $\boldsymbol{T}_{\text {th }}$ & $\boldsymbol{A}_{\text {exp }}$ & $\boldsymbol{A}_{\text {th }}$ \\
\hline 26 & 0.19 & 0.19 & 0.65 & 0.63 & 0.16 & 0.18 \\
30 & 0.18 & 0.17 & 0.67 & 0.67 & 0.16 & 0.16 \\
32 & 0.17 & 0.17 & 0.68 & 0.68 & 0.16 & 0.15 \\
34 & 0.17 & 0.17 & 0.69 & 0.68 & 0.14 & 0.15 \\
36 & 0.16 & 0.18 & 0.69 & 0.68 & 0.15 & 0.14 \\
\hline \multirow{2}{*}{ Frequency, $\mathbf{G H z}$} & $\boldsymbol{R}_{\text {exp }}$ & $\boldsymbol{R}_{\text {th }}$ & $\boldsymbol{T}_{\text {exp }}$ & $\boldsymbol{T}_{\text {th }}$ & $\boldsymbol{A}_{\text {exp }}$ & $\boldsymbol{A}_{\text {th }}$ \\
\hline 26 & 0.18 & 0.17 & 0.49 & 0.51 & 0.33 & 0.32 \\
30 & 0.15 & 0.15 & 0.51 & 0.56 & 0.34 & 0.29 \\
32 & 0.14 & 0.15 & 0.54 & 0.57 & 0.32 & 0.28 \\
34 & 0.13 & 0.15 & 0.57 & 0.58 & 0.29 & 0.28 \\
36 & 0.13 & 0.15 & 0.57 & 0.58 & 0.31 & 0.27 \\
\hline \multirow{2}{*}{ Frequency, $\mathbf{G H z}$} & \multicolumn{7}{c}{ PyC Thickness, 30 nm } & \\
\hline 26 & $\boldsymbol{R}_{\exp }$ & $\boldsymbol{R}_{\text {th }}$ & $\boldsymbol{T}_{\text {exp }}$ & $\boldsymbol{T}_{\text {th }}$ & $\boldsymbol{A}_{\text {exp }}$ & $\boldsymbol{A}_{\text {th }}$ \\
\hline 30 & 0.20 & 0.17 & 0.40 & 0.43 & 0.40 & 0.40 \\
32 & 0.16 & 0.14 & 0.41 & 0.48 & 0.43 & 0.38 \\
34 & 0.14 & 0.14 & 0.45 & 0.49 & 0.41 & 0.37 \\
36 & 0.13 & 0.14 & 0.48 & 0.50 & 0.39 & 0.36 \\
& 0.13 & 0.14 & 0.50 & 0.51 & 0.38 & 0.35 \\
\hline
\end{tabular}

The performed waveguide measurements with $110 \mathrm{~nm}$ thick film revealed (see Figure 3) that increase of frequency from $26 \mathrm{GHz}$ to $37 \mathrm{GHz}$ results in increasing of transmittance from $15 \%$ to $25 \%$ which is accompanied by decreasing of reflectivity. Similarly in "quartz + PyC" geometry, $30 \mathrm{~nm}$ thick PyC film (see Table 2) reflects $20 \%$ of EM energy at $26 \mathrm{GHz}$ and only $13 \%$ at $36 \mathrm{GHz}$. The observed frequency dispersion of transmittance and reflectance is due to the dependence of the effective incidence angle on frequency, $\cos \theta_{\text {eff }}=c K_{0} / \omega=\sqrt{1-(c \pi / \omega a)^{2}}$. This is confirmed by numerical simulation of EM wave interaction with a free standing PyC film in waveguide (see Figure 4a). It is worth noting, however, that in free space, numerical simulation of reflectance and transmittance at normal incidence for $\mathrm{PyC}$ film deposited on quartz substrate revealed almost no frequency dependence (see Figure $4 \mathrm{~b}$ ). This is because in contrast to the waveguide propagation, the characteristic interaction length in free space $l_{\sigma}=c\left(1+\sqrt{\varepsilon_{\text {quart }}}\right) / 4 \pi \sigma$ is frequency independent. Therefore in free space, the frequency dispersion can only originate from the interference caused by multiple reflections on the substrate boundaries. Since in our experiment, the substrate thickness $(0.5 \mathrm{~mm})$ is much smaller than the wavelength (about $1 \mathrm{~cm}$ ), this interference can hardly influence reflectance, absorbance and transmittance that show a weak frequency dependence (Figure $4 b$ ).

Along with conductivity and thickness, roughness can also influence the EM response of the thin film. It could be the case when both thickness and roughness are compatible with the skin depth. However one can see from Table 1 that in our experiment, both film thickness and moreover average roughness (1.25 nm for the thickest film) were much smaller than the skin depth of PyC in the $\mathrm{K}_{\mathrm{a}}$-band. 
Thus conductivity is the major parameter that influences the absorption and transmission of the studied PyC. This fact is confirmed by excellent coincidence of experimental and numerical results shown in Figure 3 and Table 2. Specifically, all experimental data in whole $\mathrm{K}_{\mathrm{a}}$-band is described by the only parameter, conductivity $\sigma=4.5 \times 10^{14} \mathrm{~s}^{-1}\left(5.0 \times 10^{4} \mathrm{~S} / \mathrm{m}\right)$, taken from the experimental data [6].

Figure 4. Frequency dependence of the reflectance, transmittance and absorbance calculated for free standing (a), and deposited on the back of the $0.5 \mathrm{~mm}$ thick quartz substrate (b), PyC film with a thickness of $110 \mathrm{~nm}$.

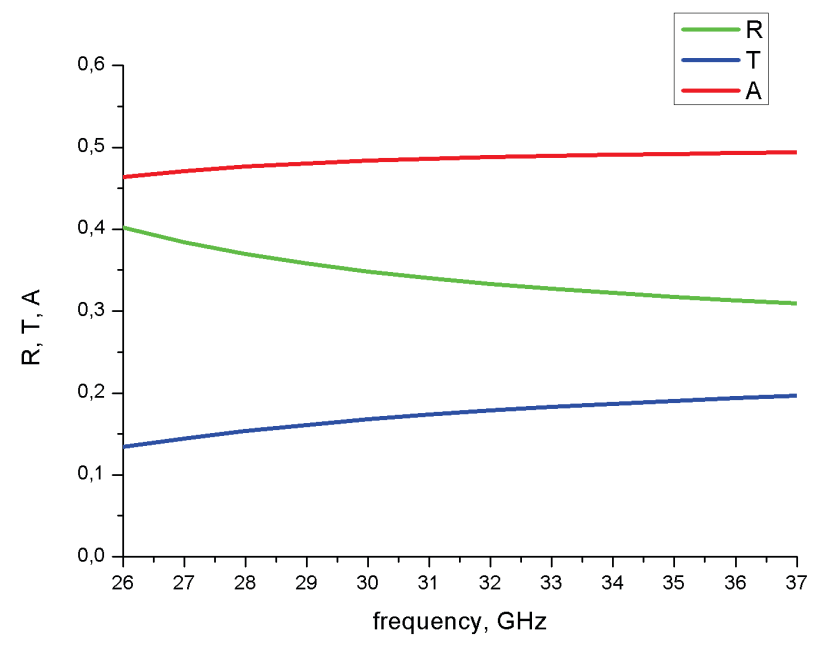

(a)

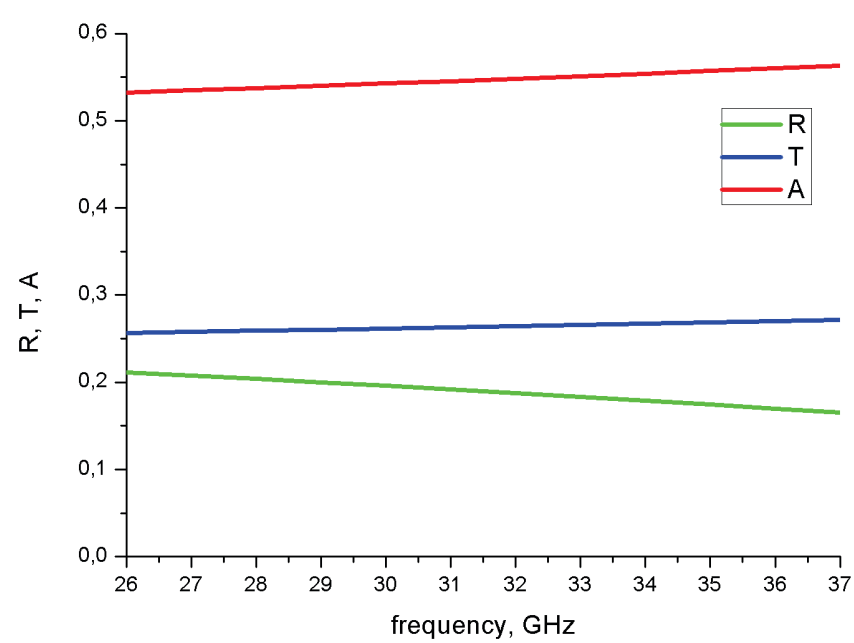

(b)

\section{Conclusions}

To conclude, we found that absorption dominates high EMI shielding efficiency of nanometrically thin PyC films. Specifically, a free standing PyC film of $110 \mathrm{~nm}$ thickness allows less than $20 \%$ of incident power to penetrate through because of $50 \%$ of absorption and more than $25 \%$ of reflection losses. This allows one to propose $\mathrm{PyC}$ films as an alternative to graphene-based EMI shields and filters $[4,9]$. At the optimal thickness, the absorption losses in the free standing film can be as high as 50\%; however, interference effects can make losses even higher in the binary structures, when the PyC film is placed onto the back of the dielectric substrate. This opens opportunities for prototyping EM devices with enhanced absorption by using PyC and other conductive ultra-thin films. It is also worth noting that the absorbance, reflectance and transmittance of the PyC films deposited on silica substrate show very weak dependence on the frequency within the $\mathrm{K}_{\mathrm{a}}$-band.

\section{Acknowledgments}

The work was partially supported by the Finnish Technology and Innovation Agency (TEKES), the EU FP7 projects CACOMEL FP7-247007 and FP7-PEOPLE-2013-IRSES-610875 NAmiceMC.

\section{Author Contributions}

Samples of PyC films were fabricated by Tommi Kaplas and Yuri Svirko. The thickness characterization was done by Tommi Kaplas and Alesya Paddubskaya. MW measurements was done 
by Alesya Paddubskaya and Polina Kuzhir. Modeling of the EM properties of pyrocarbon films on dielectric substrate was performed by Sofia Voronovich and Konstantin Batrakov. Data analysis was done by Polina Kuzhir, Konstantin Batrakov, Yuri Svirko and Sergey Maksimenko. Manuscript was written by Sofia Voronovich, Konstantin Batrakov, Polina Kuzhir and Yuri Svirko. Polina Kuzhir, Konstantin Batrakov, Sergey Maksimenko and Yuri Svirko take part in the task statement.

\section{Conflicts of Interest}

The authors declare no conflict of interest.

\section{References}

1. Ott, H. Electromagnetic Compatibility Engineering; John Wiley \& Sons: New York, NY, USA, 2009; p. 872.

2. Bae, S.; Kim, H.; Lee, Y.; Xu, X.; Park, J.-S.; Zheng, Y.; Balakrishnan, J.; Lei, T.; Kim, H.R.; Song, Y.I.; et al. Roll-to-roll production of 30-inch graphene films for transparent electrodes. Nat. Nanotechnol. 2010, 5, 574-578.

3. Hong, S.K.; Kim, K.Y.; Kim, T.Y.; Kim, J.H.; Park, S.W.; Kim, J.H.; Cho, B.J. Electromagnetic interference shielding effectiveness of monolayer graphene. Nanotechnology 2012, 23, 5.

4. Stankovich, S.; Dikin, D.A.; Dommett, G.H.B.; Kohlhaas, K.M.; Zimney, E.J.; Stach, E.A.; Piner, R.D.; Nguyen, S.B.T.; Ruoff, R.S. Graphene-based composite materials. Nature 2006, 442, 282-286.

5. Kuzhir, P.; Paddubskaya, A.; Maksimenko, S.; Kaplas, T.; Svirko, Y. Microwave absorption properties of pyrolytic carbon nanofilm. Nanoscale Res. Lett. 2013, 8, 6.

6. Kaplas, T.; Svirko, Y. Direct deposition of semitransparent conducting pyrolytic carbon films. J. Nanophotonics 2012, 6, 7.

7. Batrakov, K.; Kuzhir, P.; Maksimenko, S.; Paddubskaya, A.; Voronovich, S.; Kaplas, T.; Svirko, Yu. Enhanced microwave shielding effectiveness of ultrathin pyrolytic carbon films. Appl. Phys. Lett. 2013, 103, 4.

8. Benzinger, W.; Becker, A.; Hüttinge, K. Chemistry and kinetics of chemical vapor deposition of pyrocarbon: I. Fundamentals of kinetics and chemical reaction engineering. Carbon 1996, 34, 957-966.

9. Kuzhir, P.; Volynets, N.; Maksimenko, S.; Kaplas, T.; Svirko, Yu. Multilayered graphene in $\mathrm{K}_{\mathrm{a}}$-band: Nanoscale coating for aerospace applications. J. Nanosci. Nanotechnol. 2013, 13, 5864-5867.

(C) 2014 by the authors; licensee MDPI, Basel, Switzerland. This article is an open access article distributed under the terms and conditions of the Creative Commons Attribution license (http://creativecommons.org/licenses/by/3.0/). 